\title{
Comunicação
}

[Communication]

\section{Parasitoides de dípteros coletados em excrementos de animais no sul de Goiás}

[Parasitoids of dipterous collected in animal excrements in Southern Goiás, Brazil]

\author{
C.H. Marchiori ${ }^{1}$, L.F. Barbaresco ${ }^{2}$, M. E. Ferreira ${ }^{2}$ \\ ${ }^{1}$ Instituto Federal de Educação e Tecnologia Goiano \\ Caixa Postal 50 \\ 74000-03 - Goiânia, GO \\ ${ }^{2}$ Instituto Luterano de Ensino Superior de Itumbiara - ULBRA - Itumbiara, GO
}

Além dos inseticidas, também podem ser usados como controle dos insetos os chamados reguladores naturais tanto na agricultura como em áreas de criação animal. O controle químico de insetos em ambiente urbano e rural é dificultado devido ao perigo de contaminação do homem, animais e ambiente. Portanto, o controle biológico de moscas com uso de microimenópteros parasitoides constitui uma alternativa para o problema, por ser um método seguro, de fácil manuseio e baixo custo (Silveira et al., 1989; Marchiori et al., 2001; Carvalho et al., 2003). Assim, o objetivo deste estudo foi verificar as espécies de parasitoides associadas a dipteros em fezes de bovinos e búfalos e fezes de galinha, coletados no sul de Goiás.

Quinzenalmente, 10 placas de fezes ( de bovinos e de búfalos) frescas foram marcadas imediatamente após sua emissão, em pastagens de braquiária (Brachiaria brizantha), com auxílio de estacas de madeira branca $(30 \mathrm{~cm}$ de altura e $5 \mathrm{~cm}$ de espessura), para determinação precisa do tempo entre a emissão e a coleta, permaneceram no campo por 15 dias. Sete amostras foram colhidas e levadas ao laboratório para a extração das pupas pelo método da flutuação. Juntamente com as fezes, foram retirados $5 \mathrm{~cm}$ do substrato situado abaixo do local de deposição no solo. As pupas, retiradas com o auxílio de uma peneira, foram contadas e individualizadas em cápsulas de gelatina (número 00) até a emergência das moscas e/ou dos parasitoides. Os parasitoides e as moscas emergidos, identificados com auxílio de um microscópio estereoscópio, foram conservados em álcool $70 \%$. O experimento foi realizado no período de maio de 2003 a junho de 2004.

O experimento com fezes de galinha foi realizado na granja da Chácara Casa do Campo, em Itumbiara $\left(18^{\circ} 25^{\prime} \mathrm{S}\right.$ e $\left.49^{\circ} 13^{\prime} \mathrm{W}\right)$, Goiás. As fezes, provenientes de 40 aves criadas em gaiolas, variaram de umidade, de pastosa a firme. As fezes frescas (pastosa), coletadas imediatamente após a emissão, e colocadas em cinco bacias de $30 \mathrm{~cm}$ de diâmetro por $12 \mathrm{~cm}$ de altura, foram deixadas no próprio ambiente, em local seco, por 15 dias, para a extração das pupas pelo método da flutuação. Os parasitoides e as moscas emergidos foram identificados com auxílio de um microscópio estereoscópio. O experimento foi realizado de abril de 2006 a março de 2007.

A porcentagem de parasitismo foi calculada pelo número de pupas parasitadas/ número total de pupas coletadas x 100. A hipótese de atração dos parasitoides pelos seus hospedeiros foi testada pelo qui-quadrado.

Foram coletados 78 espécimes de parasitoides (Tab. 1) de 628 pupas de dípteros das fezes bovinas e 310 parasitoides de 3116 pupas de dípteros das fezes de galinha. Provavelmente, essa diferença no número de parasitoides e de moscas coletadas nos dois substratos deve-se à maior oferta de alimento (ração para aves).

Recebido em 21 de novembro de 2008 
Tabela 1. Micro-himenópteros parasitoides coletados em fezes de bovinos e fezes de galinha no sul de Goiás

\begin{tabular}{lcc} 
Grupo taxonômico & $\begin{array}{c}\text { Número de indivíduos } \\
\text { em fezes bovinas }\end{array}$ & $\begin{array}{c}\text { Número de indivíduos } \\
\text { em fezes de galinha }\end{array}$ \\
\hline Hymenoptera & & \\
Braconidae & 0 & 1 \\
Aphaereta sp. & 20 & \\
Gnathopleura quadridentata & & 0 \\
Diapriidae & 1 & \\
Trichopria sp. & & 10 \\
Encyrtidae & 0 & \\
Taechinaephagus zealandicus & & 2 \\
Eurytomidae & 0 & 0 \\
Eurytoma sp. & & 0 \\
Figitidae & 2 & 0 \\
Kleidotoma nigra & 2 & 0 \\
Paraganaspis egeria & 2 & \\
Triplasta atrocoxalis & 8 & 12 \\
Triplasta coxalis & & 65 \\
Pteromalidae & 0 & 38 \\
Nasonia vitripennis & 5 & 1 \\
Pachycrepoideus vindemmiae & 4 & 3 \\
Spalangia cameroni & 7 & 10 \\
Spalangia drosophilae & 2 & 21 \\
Spalangia endius & 7 & 310 \\
Spalangia nigra & 18 & \\
Spalangia nigroaenea & 0 & \\
Spalangia sp. & 78 & \\
Total & & \\
\hline & & \\
\hline
\end{tabular}

As espécies mais frequentes nas fezes bovinas e de galinha foram: Gnathopleura quadridentata Wharton (Hymenoptera: Braconidae), com $25,6 \%$ e Spalangia endius walker (Hymenoptera: Pteromalidae) com $47,7 \%$, respectivamente. A capacidade de busca do parasitoide, a sazonalidade, a maior presença de seus hospedeiros na área de coleta e o seu maior potencial competitivo na fase larval podem explicar a maior frequência dessas espécies, apesar de não ter sido testado nenhum fator para explicar a diferença na frequência dos parasitoides neste trabalho.

G. quadridentata demonstra atração por muscoides, especialmente da família Sarcophagidae. Essa espécie comporta-se como parasitoide solitário que emerge do pupário de seus hospedeiros (Wharton, 1979). Segundo Boucek (1963) e Legner et al. (1976), esse parasitoide é cosmopolita, e é capaz de atacar pupas de Calliphoridae, Sarcophagidae, Muscidae e Tephritidae.

A porcentagem de parasitismo observada nas fezes bovinas e de galinha foi de $12,4 \%$ e $10,0 \%$, respectivamente. A maior porcentagem de parasitismo nas fezes bovinas foi a do parasitoide G. quadridentata, $28,6 \%$, e nas fezes de galinha a do parasitoide $S$. endius, 7,2\%, provavelmente devido à maior afinidade pelas espécies hospedeiras presentes, apesar de não ter sido testado nenhum fator para explicar a diferença na porcentagem de parasitismo dos parasitoides (Tab. 2 e 3). 


\section{Marchiori et al.}

Tabela 2. Prevalência de micro-himenópteros parasitoides coletados em fezes de bovinos no sul de Goiás, no período de maio de 2003 a junho de 2004

\begin{tabular}{|c|c|c|c|c|}
\hline Espécie de dípteros & $\begin{array}{l}\text { Número de } \\
\text { indivíduos }\end{array}$ & *Parasitoide & $\begin{array}{l}\text { Número de } \\
\text { indivíduos }\end{array}$ & Porcentagem \\
\hline Archisepsis scabra & 40 & Spalangia drosophilae & 04 & 10,0 \\
\hline \multirow[t]{2}{*}{ Brontaea debilis } & \multirow[t]{2}{*}{56} & Spalangia cameroni & 01 & 1,79 \\
\hline & & Spalangia nigroaenea & 02 & 3,57 \\
\hline \multirow[t]{5}{*}{ Brontaea quadristigma } & \multirow[t]{5}{*}{49} & Kleidotoma nigra & 02 & 4,08 \\
\hline & & Spalangia cameroni & 01 & 2,04 \\
\hline & & Spalangia drosophilae & 01 & 2,04 \\
\hline & & Spalangia. endius & 01 & 2,04 \\
\hline & & Spalangia nigroaenea & 05 & 10,2 \\
\hline \multirow[t]{2}{*}{ Cyrtoneurina pararescita } & \multirow[t]{2}{*}{151} & Spalangia nigra & 03 & 1,99 \\
\hline & & Spalangia nigroaenea & 05 & 3,31 \\
\hline Musca domestica & 10 & ausente & 01 & 10,0 \\
\hline Oxysarcodexia thornax & 70 & Spalangia cameroni & 20 & 28,6 \\
\hline \multirow[t]{6}{*}{ Palaeosepsis spp. } & \multirow[t]{6}{*}{107} & Gnathopleura quadridentata & 02 & 1,87 \\
\hline & & Paraganaspis egeria & 02 & 1,87 \\
\hline & & Spalangia drosophilae & 01 & 0,93 \\
\hline & & Spalangia endius & 02 & 1,87 \\
\hline & & Triplasta atrocoxalis & 08 & 7,48 \\
\hline & & Triplasta coxalis & 01 & 0,93 \\
\hline \multirow[t]{4}{*}{ Ravinia belforti } & \multirow[t]{4}{*}{63} & Trichopria sp. & 05 & 7,94 \\
\hline & & Pachycrepoideus vindemmiae & 01 & 1,59 \\
\hline & & Spalangia cameroni & 04 & 6,35 \\
\hline & & Spalangia nigra & 06 & 9,52 \\
\hline Outras espécies não parasitadas & 82 & - & - & - \\
\hline Total de pupas 628 & & & 78 & 12,4 \\
\hline
\end{tabular}

Tabela 3. Parasitoides de dípteros sinantrópicos coletados em fezes de galinhas poedeiras em Itumbiara, Goiás, Brasil

\begin{tabular}{|c|c|c|c|c|}
\hline Grupo taxonômico & $\begin{array}{c}\mathrm{N}^{\circ} \text { de } \\
\text { indivíduos }\end{array}$ & Espécie & $\begin{array}{l}\mathrm{N}^{\circ} \text { de pupas } \\
\text { parasitadas }\end{array}$ & Porcentagem \\
\hline \multicolumn{5}{|l|}{ Fanniidae } \\
\hline \multirow[t]{2}{*}{ Fannia pusio } & 153 & Eurytoma sp. & 2 & 1,3 \\
\hline & & Pachycrepoideus vindemmiae & 9 & 5,9 \\
\hline \multicolumn{5}{|l|}{ Muscidae } \\
\hline \multirow{10}{*}{ Musca domestica } & 1982 & Aphaereta sp. & 1 & 0,1 \\
\hline & & Nasonia vitripennis & 11 & 0,6 \\
\hline & & Pachycrepoideus vindemmiae & 52 & 2,6 \\
\hline & & Spalangia cameroni & 38 & 1,9 \\
\hline & & Spalangia drosophilae & 1 & 0,1 \\
\hline & & Spalangia endius & 143 & 7,2 \\
\hline & & Spalangia nigra & 3 & 0,2 \\
\hline & & Spalangia nigroaenea & 10 & 0,5 \\
\hline & & Spalangia sp. & 21 & 1,1 \\
\hline & & Tachinaephagus zealandicus & 1 & 0,1 \\
\hline \multirow[t]{2}{*}{ Ophyra sp. } & 529 & Nasonia vitripennis & 1 & 0,2 \\
\hline & & Spalangia endius & 4 & 0,8 \\
\hline \multicolumn{5}{|l|}{ Sarcophagidae } \\
\hline \multirow{2}{*}{ Sarcodexia lambens } & 222 & Pachycrepoideus vindemmiae & 2 & 0,9 \\
\hline & & Tachinaephagus zealandicus & 9 & 4,1 \\
\hline \multicolumn{5}{|l|}{ Syrphidae } \\
\hline Ornidia obesa & 226 & Pachycrepoideus vindemmiae & 2 & 0,9 \\
\hline $\begin{array}{ll}\begin{array}{l}\text { Outras espécies } \\
\text { parasitadas }\end{array} & \text { não } \\
\end{array}$ & 04 & - & - & - \\
\hline Total & 3116 & - & 310 & - \\
\hline
\end{tabular}


Com relação à atração dos hospedeiros pelos seus parasitoides nas fezes bovinas, $G$. quadridentata apresentou atração por Oxysarcodexia thornax (Walker) (Diptera: Sarcophagidae); Kleidotoma nigra (Hartig) (Hymenoptera: Figitidae) atração por Brontaea quadristigma (Thomson) (Diptera: Muscidae); Pachycrepoideus vimdemmiae (Rondani) (Hymenoptera: Pteromalidae) apresentou atração por Ravinia belforti (Prado \& Fonseca) (Diptera: Sarcophagidae); Paraganaspis egeria Díaz et al. (Hymenoptera: Figitidae) atração por Palaeosepsis spp. (Diptera; Sepsidae); Spalangia cameroni Perkins (Hymenoptera: Pteromalidae) atração por Brontaea debilis (Williston) (Diptera: Muscidae), por B. quadristigma, por $M$. domestica e por $R$. belforti; $S$. drosophilae atração por Archisepsis scabra (Loew) (Diptera: Sepsidae), por $B$. quadristigma e por Palaeosepsis spp.; Spalangia endius Walker (Hymenoptera: Pteromalidae) atração por $B$. quadristigma e por Palaeosepsis spp.; S. nigra atração por Cyrtoneurina pararescita Couri (Diptera: Muscidae) e por $R$. belforti; $S$. nigroaenea atração por $B$. debilis, por $B$. quadristigma, por $C$. pararescita e por $R$. belforti; Trichopria sp. (Hymenoptera: Diapriidae) atração por Palaeosepsis spp.; Triplasta atrocoxalis (Ashmead) (Hymenoptera; Figitidae) atração por Palaeosepsis spp.; e Triplasta coxalis (Ashmead) (Hymenoptera;
Figitidae $)$ atração por Palaeosepsis spp. $\left(\chi^{2}=\right.$ 250,91; GL=77; $\mathrm{P}<0,05)$.

Com relação à atração dos hospedeiros pelos seus parasitoides nas fezes de galinha, Aphareta sp. (Hymenoptera: Braconidae) apresentou atração por Musca domestica L.; Eurytoma sp. atração por Fannia pusio (Wiedemann) (Diptera: Fanniidae); Nasonia vitripennis (Walker) atração por M. domestica e Ophyra sp. (Diptera: Muscidae); $P$. vimdemmiae atração por $F$. pusio, M. domestica e Ornidia obesa Fabricius (Diptera: Syrphidae); S. cameroni atração por $M$. domestica; S. drosophilae atração por $M$. domestica; S. endius atração por $M$. domestica e Ophyra sp.; S. nigra, S. nigroaenae e Spalangia sp. atração por M. domestica; e Taechinaephagus zealandicus (Ashmead) (Hymenoptera: Encyrtidae) atração por $F$. pusio e por Sarcodexia lambens (Wiedemann) (Diptera: Sarcophagidae) $\left(\chi^{2}=235,17\right.$; GL: $\left.40 ; \mathrm{P}<0,05\right)$.

Reguladores naturais, como os parasitoides, podem ser usados no controle biológico de moscas. Esses agentes são responsáveis pela redução de populações de moscas sinantrópicas.

Palavras-chave: Diptera, Hymenoptera, inimigo natural, controle biológico, fezes

\begin{abstract}
This study determined the species of microhymenopterous of Diptera present in cattle and buffalo dung, from May 2003 to June 2004 and from April 2006 to March 2007 in chicken manure. All samples were collected in Southern Goiás State, Brazil. The dipterous pupae were obtained by the flotation method. They were individually placed in gelatin capsules until the emergence of the flies and/or their parasitoids. The percentages of parasitism in cattle dung and chicken manure were $12.4 \%$ and $10.0 \%$, respectively.
\end{abstract}

Keywords: Diptera, Hymenoptera, natural enemy, biocontrol, manure

\section{REFERÊNCIAS BIBLIOGRÁFICAS}

CARVALHO, A.R.; MELLO, R.P.; D’ALMEIDA, J.M. Micro-himenópteros parasitoides de Chrysomya megacephala. Rev. Saúde Pública, v.37, p.810-812, 2003.

BOUCEK, Z. A taxonomic study in Spalangia Latr. (Hymenoptera: Chalcidoidea). Acta. Entomol. Mus. Nat. Pragae, v.35, p.429-512, 1963.

LEGNER, E.E.; MOORE, I.; OLTON, G.S. Tabular keys \& biological notes to commom parasitoids of synanthropic Diptera breeding in accumulated animal wastes. Entomol. News, v.87, p.113-144, 1976.
MARCHIORI, C.H.; OLIVEIRA, A.T.; LINHARES, A.X. Artrópodes associados a massas fecais bovinas no sul do Estado de Goiás. Neotrop. Entomol., v.30, p.19-24, 2001.

SILVEIRA, G.A.R.; MADEIRA, N.G.; AZEREDOESPIN, A.M. et al. Levantamento de microhimenópteros parasitoides de dípteros de importância médicoveterinária no Brasil. Mem. Inst. Oswaldo Cruz, v.84, p.505-510, 1989.

WHARTON, R.A. Puparia of cyclorrhaphous Diptera from bovine dung in open pasture and rangeland in the transition zone of Western North America. Ann. Entomol. Soc. Am., v.72, p.80-89, 1979. 